\title{
A Tracking Method in FM Broadcast- based Passive Radar Systems
}

\author{
Ádám $\mathrm{KISS}^{1 \oplus}$ - Levente DUDÁS²
}

\begin{abstract}
Passive radars are popular because without the expensive, high-power-rated $R F$ components, they are much cheaper than the active ones, nevertheless, they are much harder to detect from their electromagnetic emission. Passive radars produce so-called $R V$ matrices in an intermediate signal processing step. Although accurate $R V$ matrices are found in DVB-T-based passive radars, the characteristics of the FM signals are not always suitable for this purpose. ${ }^{3}$ In those situations, further signal processing causes false alarms and unreliable plots, misleads the tracker, and consumes power for processing unnecessarily, which matters in portable setups. Passive radars also come with the advantage of a possible MIMO setup, when multiple signal sources (broadcast services for example) are reflected by multiple targets to the receiver unit. One common case is the stealth aircraft's which form is designed to reflect the radar signal away from the active radar, but it could also reflect the signals of the available broadcast channels. Only one of these reflected signals could reveal the position of the target.
\end{abstract}

Keywords: Passive radar, bistatic tracking, FM passive radar, RV matrix

\section{Introduction to the test system}

Our instrument starts with a four-element Yagi array. One of the antennas points to a publicly known broadcast FM transmitter tower, the others point to the opposite direction and form a three-element MRA, a linearly distributed antenna array with minimal redundancy. ${ }^{4}$ All of the four antennas are connected to a coherent four-channel software-defined radio.

MSc student, Budapest University of Technology and Economics Department of Broadband Infocommunication and Electromagnetic Theory, Radar Research Group; e-mail: kiss.adam@simonyi.bme.hu

2 Lecturer, Budapest University of Technology and Economics Department of Broadband Infocommunication and Electromagnetic Theory, Radar Research Group; e-mail: dudas.levente@vik.bme.hu

3 Rudolf Seller, Tamás Pető, Levente Dudás and Levente Kovács, 'Passzív radar I. rész', Haditechnika 53, 6 (2019), 51-55.

$4 \quad$ Li Bo and Sun Chao, 'A new method for array extension based on minimum redundancy linear array', IEEE 10th International Conference on Signal Processing Proceedings, Beijing, 2010, 332-335. 
In the first step of the signal processing, the reference signal is subtracted from the surveillance channels, which is done by a Wiener filter. Having the pure surveillance signal, the reference channels can be cross-correlated with the surveillance ones.

$$
\chi\left(\tau, f_{d}\right)=\left\langle s_{s}(t), s_{r e f}(t-\tau) e^{j 2 \pi f_{d} t}\right\rangle=\int_{-\infty}^{\infty} s_{s}(t) s_{r e f}^{*}(t-\tau) e^{-j 2 \pi f_{d} t} d t \approx \int_{0}^{T} s_{s}(t) s_{r e f}^{*}(t-\tau) e^{-j 2 \pi f_{d} t} d t
$$

where $\chi\left(\tau, f_{d}\right)$ is the RV matrix, $\tau$ is the time delay between the signal of the surveillance channel $\left(s_{s}\right)$ and the reference channel $\left(s_{r e f}\right)$, and $f_{d}$ is the Doppler shift caused by the movement of the target, and $T$ is the time interval during samples are gathered.

In the used configuration the dimensions of the RV matrix were $75 \mathrm{~km}$ and, $\pm 955 \mathrm{~m} / \mathrm{s}$, the resolution was 64 cells both in velocity and in distance.

Only a signal with an infinite spectrum could produce a Dirac delta as its autocorrelation function. According to the scaling theorem, the more the bandwidth of a signal is decreased, the wider its autocorrelation peak. In FM systems this means when the modulating signal (which includes stereo pilot and the RDS carrier among others) has lower energy, lower effective frequency deviation is produced during the modulation, hence the momentary spectrum is narrower, the autocorrelation peak is wider. The broadcast stations often use compressors on the transmitter side, so this case is rare.

The FM modulation also has a special property that shows itself when modulating with pure sine waves. In this case, the modulated spectrum is discrete despite the non-linearity of the modulation. All these unique, randomly appearing cases decrease the randomness of the signal, resulting in a time-variant autocorrelation function in general.

Due to the fluctuation of the FM broadcast signal's autocorrelation properties, sometimes the RV matrix is not as clean as should be for accurate processing, so the unusable ones should be dropped.

\section{Method of selection}

Finding one algorithm to work properly for every case is difficult, a more convenient and more easily understandable/developable solution is diverse, and sums up the results of different scenarios.

\section{Wide autocorrelation}

The autocorrelation function of the recorded sample may be too wide, not as narrow as it could be suggested from the bandwidth supposing the necessary randomness. In this case, the detection of the peak could be impossible. To separate these cases, checking against the distribution could be used. The method assigns a scalar to every point of the RV matrix, in this test case, that was the absolute value function. The challenge is to find the number of elements in every row having a greater metric than a specified threshold. The threshold can be chosen by a constant between 0 and 1, representing a linear scale between the minimum and the maximum metric found in the matrix. Knowing the number of columns 
above the threshold in a row can reveal if there is a wide correlation peak with the Doppler shift pointed by the row number. The minimum width of a correlation peak failing the test should be chosen. In the test, the multiplied value of the matrix width and the previously mentioned threshold constant between 0 and 1 was used.

A matrix can be found faulty by the number of failed lines in it. In the examples below, the threshold value of 0.43 was used. Due to the choice of the threshold metric, this test is called dynamic check in this paper, since the metric is calculated from the dynamic of the RV row.

\section{Cow-like RV matrix}

It is also possible that the incoming matrix has many so-called patches in it. This is caused by multiple peaks in the autocorrelation function. One algorithm to detect these cases is to map every cell in the RV matrix as a graph, give a metric to all the adjacent cells, then find the shortest path from one dedicated point to another. Using the same endpoints shows the difference between the RV matrices in this view.

In this test setup, a one-dimensional Manhattan metric was given to every edge adjacent cell, so the metric is the positive difference of the difference between the cells' absolute values:

$$
W_{i, j}=\| \chi_{i}|-| \chi_{j}||
$$

where $\chi$ is the RV matrix, $i$ and $j$ are cells from the RV matrix. Then a Dijkstra algorithm ${ }^{5}$ can be performed on the given matrix, which gives the shortest path between the start and the stop points by building deeper and deeper distance trees from a start point. For the start $(3,3)$ point was used from the top left corner (nearest distance and the biggest velocity moving away), and $(61,61)$ was used as a destination. Picking these is better, than the corners themselves, since that would decrease the degree of freedom for the algorithm. The upper limit used in the tests was 110 for the pieces of the shortest path, and 20 for the summed weights along the path.

An example of cow-like RV matrices and three reference matrices are shown in Figure 1 and Figure 2. The data registration was taken in early 2020 at Budapest Ferenc Liszt International Airport.

Chen Peng, Xiaoqing Lu, Jiyang Dai and Linfei Yin, 'Research of path planning method based on the improved Voronoi diagram’, 25th Chinese Control and Decision Conference (CCDC), Guiyang, 2013, 2940-2944. 


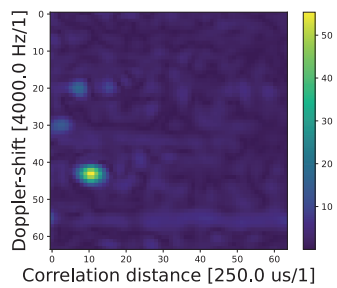

(a) RV matrix from the first antenna of the MRA

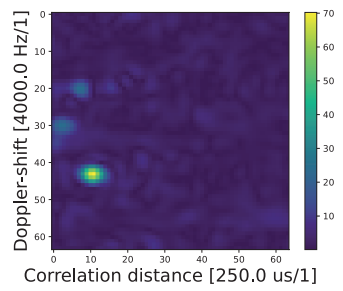

(b) RV matrix from the second antenna of the MRA

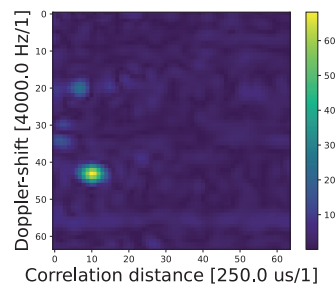

(c) RV matrix from the third antenna of the MRA

\begin{tabular}{lll} 
Antenna & Property & Result \\
\hline First & Number of Dijkstra optimal path elements & 102 \\
First & Length of Dijkstra optimal path & 22.02 \\
First & Failed lines on dynamic check & 0 \\
Second & Number of Dijkstra optimal path elements & 105 \\
Second & Length of Dijkstra optimal path & 21.08 \\
Second & Failed lines on dynamic check & 0 \\
Third & Number of Dijkstra optimal path elements & 105 \\
Third & Length of Dijkstra optimal path & 20.43 \\
Third & Failed lines on dynamic check & 0 \\
\hline & Summary & Passed
\end{tabular}

Figure 1: Passed result of the sanity checking on a set of RV matrices from the MRA

Source: The results of the algorithms of the measurement data introduced in the text.

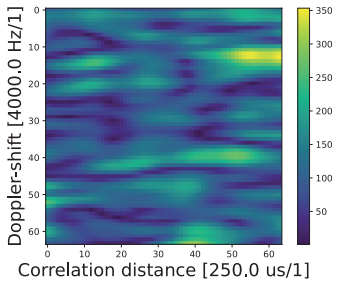

(a) RV matrix from the first antenna of the MRA

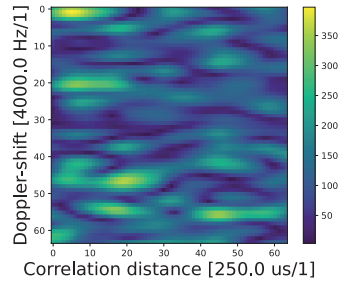

(b) RV matrix from the second antenna of the MRA

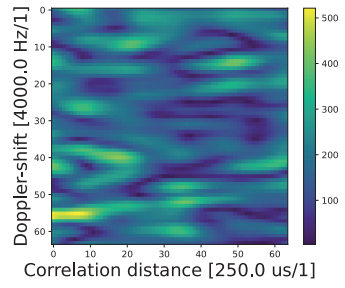

(c) RV matrix from the third antenna of the MRA

\begin{tabular}{lll} 
Antenna & Property & Result \\
\hline First & Number of Dijkstra optimal path elements & 133 \\
First & Length of Dijkstra optimal path & 754.84 \\
First & Failed lines on dynamic check & 9 \\
Second & Number of Dijkstra optimal path elements & 126 \\
Second & Length of Dijkstra optimal path & 1057.66 \\
Second & Failed lines on dynamic check & 7 \\
Third & Number of Dijkstra optimal path elements & 137 \\
Third & Length of Dijkstra optimal path & 1269.56 \\
Third & Failed lines on dynamic check & 15 \\
\hline & Summary & Failed
\end{tabular}

Figure 2: Failed result of the sanity checking on a set of $R V$ matrices from the MRA

Source: The results of the algorithms of the measurement data introduced in the text. 


\section{Summing up the results}

The Dijkstra algorithm appeared to be more reliable in determining the sanity level of a matrix, but not all the bad matrices reached the decision thresholds. Every failed test for a matrix was counted as one point. If there was a failed Dijkstra algorithm during the inspection of the MRA in the view of the weighted path length, one plus point was added to the sum of the three matrices' points. When an MRA set had five or more points, it was dropped in the test setup.

\section{Hit generation}

Having sane RV matrices the potentially target-related cells could be determined. These are called hits. The hits considered to be a target are called plots.

Even on the friendly RV matrices, finding the hits on them is still a challenge. Although the cell-averaging CFAR algorithm is commonly used for this problem, in this test system a new kind of implementation was used.

\section{Theory of operation}

A Wiener filter is used at the very beginning of the signal processing chain, hence it is known that the zero Doppler line of the RV matrix is approximately zero. The algorithm:

1. Reinterpret the matrix cells as a set of numbers named $S$.

2. Throw away the highest and lowest $h$ and $l$ per cent of the elements ordered by their absolute value from $S$.

3. Determine a threshold level from the remaining numbers' absolute value.

4. Having determined the threshold, select every point with higher absolute values than the threshold.

5. Select the potential hits from points above the threshold.

6. Accumulate the results from the three RV matrices and decide for hits.

In our tests, 25 per cent for $h$ and $l$ were used. Ignoring these elements allows to neglect the presence of the uncommon zero Doppler line and the ringing of the hits, assuming that a large enough $h$ and $l$ was chosen.

The remaining numbers have a mean value and a variance. The threshold was calculated by summing the mean component and the variance multiplied by an encouraging factor:

$$
T=M+(B \cdot V)
$$

where $T$ is the threshold, $M$ is the mean value, $V$ is the variance and $B$ is the encouraging factor. The encouraging factor compensates for the statistical error from the ignored elements and also contains a minimal hit-to-noise relation from where a peak in the RV matrix is considered to be a hit. In the tests, the value 50 was used for $B$. 
The hits from the points above the threshold are selected by the sign of the gradients for the absolute values in the matrix. The local maximums are hits on one RV matrix. An absolute hit is triggered when at least two of the antennas have a hit on the same RV coordinates.

Figures 3 and 4 show the result of this technique on three reference matrices and three others not having the necessary autocorrelation property. The data registration was taken in early 2020 at Budapest Ferenc Liszt International Airport.

\section{Angle measurement}

The measurement of the incoming wave is done by a three-element MRA. In the tests, the Capon method ${ }^{6}$ was used, when it failed, it was replaced by the Bartlett method. The correlation values filling the correlation matrix for the used methods are from the RV matrix fields where the hits occur.
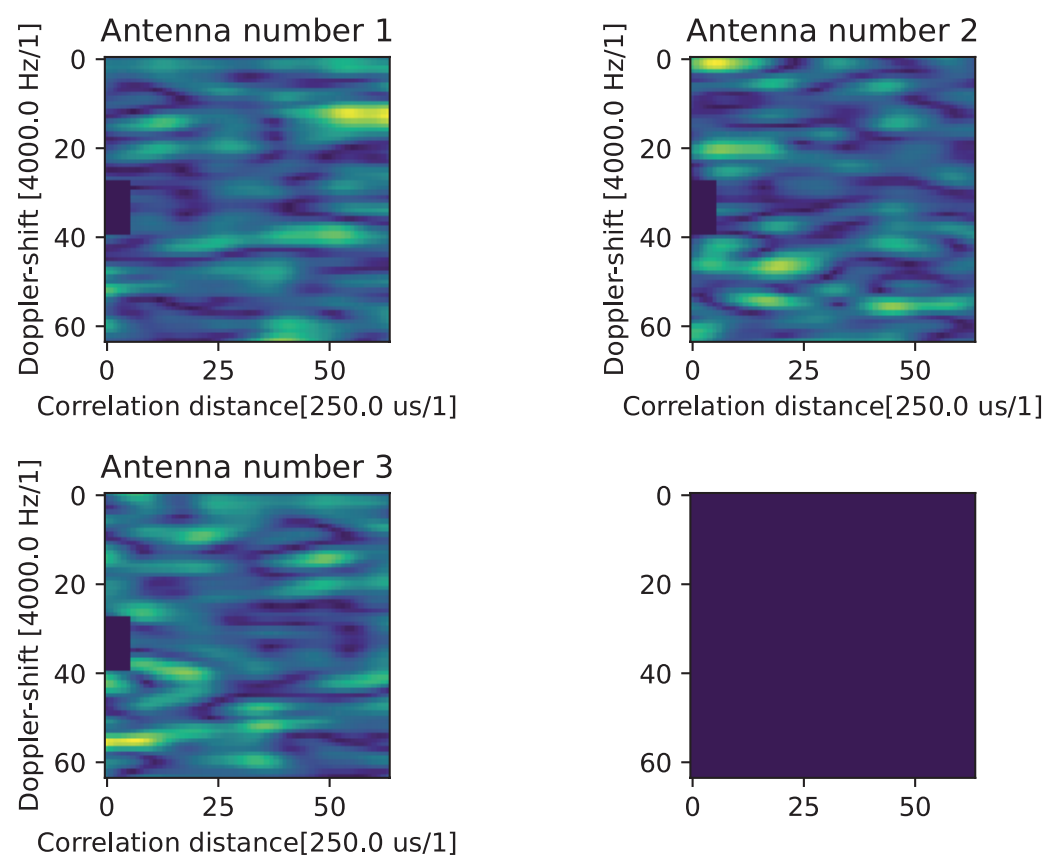

Figure 3: Hits on a measurement

Source: The results of the algorithms of the measurement data introduced in the text.

Michal Meller and Kamil Stawiarski, 'Capon-like DoA estimator for rotating arrays', 2019 International Radar Conference (RADAR), Toulon, 2019, 1-6. 

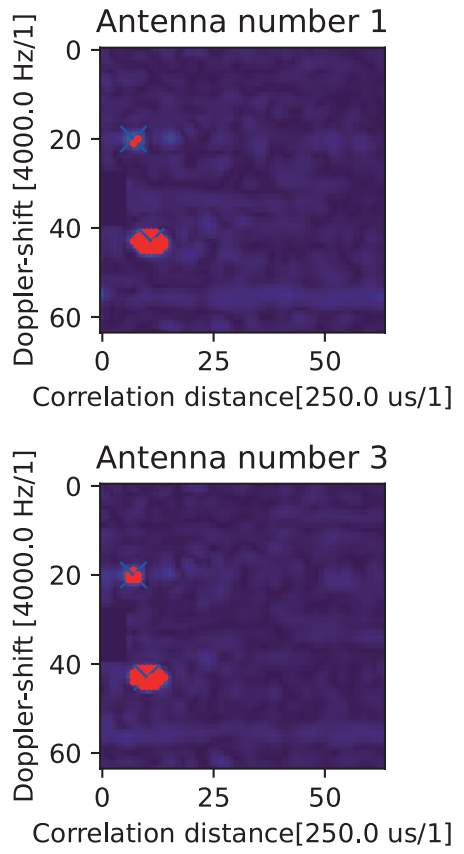
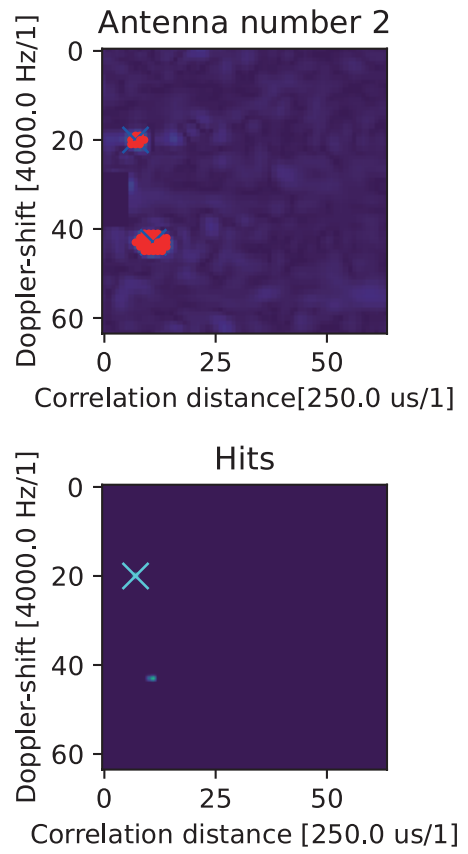

Figure 4: Hits on a measurement

Source: The results of the algorithms of the measurement data introduced in the text.

\section{Correction method}

The calculation of every Doppler-corrected reflection is an FFT spectrum. The used windowing function is a rectangular one, which produces the narrowest main lobe in the spectrum, but high-level sidelobes. The task from this point is analogous to a frequency measurement in a basic Fourier spectrum.

The method used in the article resamples a basic sinc lobe (seen in an ideal discrete domain Fourier spectrum) with the same sample rate, but with different shifts between the original bins and the output. The produced three Fourier-bin-wide vector is then correlated with the highest energy row in the RV matrix around the potential target. Where the correlation is the highest, the shift is nearly equal to the distance of the theoretical direct received signal and the test signal peak. The Gaussian noise in the matrix causes a little uncertainty, and so does the width of the FM signal's correlation. The tracker can treat normal distribution noise in the next stages, the goal of this method is to normalise the distribution of the measures. The necessary plus information comes from the adjacent cells of the RV matrix. 
Resampled sinc function in the test system:

$$
f[n, \text { offset }]=\left|\frac{\sin (\pi(n+(\text { offset } / N)))}{\pi(n+(\text { offset } / N))}\right|
$$

where $N$ is the precision of this estimation process, the number of steps in the estimation process; $n$ is the distance from the point with the highest energy; and offset is the running index from which the offset is calculated. The offset variable iterates from $-N$ to $N$. The absolute value function is needed, because, in this version of the algorithm only the amplitude of the RV matrix is correlated, not its complex value.

The correlation is given by the traditional dot product of two vectors: one is from the proper row of the RV matrix, the other is $f[n, o f f s e t]$, where $n$ is the dimension of the scanning test vector, and offset is the currently indexed phase delay in the resampling. The task is to determine the offset value at the maximum value of this correlation.

In the tests, value 3 was used for $n$, and value 31 was used as steps for $N$.

Having the offsets for the three RV matrices, they were averaged to estimate the real offset.

Filter output on a sample track is shown in Table 1.

As it can be seen from the test result, by using the adjacent cells and a sinc-based filter, a less quantised bistatic distance estimation can be made than without this method.

Table 1: Filter result on a recording from a landing plane

\begin{tabular}{|c|c|c|c|c|}
\hline $\begin{array}{c}\text { Serial number of } \\
\text { the RV matrix }\end{array}$ & $\begin{array}{c}\text { Matrix row with } \\
\text { the highest value }\end{array}$ & $\begin{array}{c}\text { Estimated } \\
\text { Doppler } \\
\text { coordinate }\end{array}$ & $\begin{array}{c}\text { Matrix column } \\
\text { with the highest } \\
\text { value }\end{array}$ & $\begin{array}{c}\text { Estimated } \\
\text { distance } \\
\text { coordinate }\end{array}$ \\
\hline 6 & 47 & 46.69 & 18 & 19.00 \\
\hline 8 & 47 & 46.58 & 18 & 18.49 \\
\hline 11 & 46 & 46.51 & 16 & 15.82 \\
\hline 15 & 45 & 45.58 & 15 & 15.31 \\
\hline 16 & 45 & 44.69 & 15 & 15.64 \\
\hline 17 & 45 & 44.51 & 15 & 14.78 \\
\hline 20 & 45 & 45.62 & 14 & 14.84 \\
\hline 21 & 45 & 45.82 & 14 & 14.18 \\
\hline 23 & 45 & 45.64 & 13 & 13.33 \\
\hline 27 & 45 & 45.11 & 12 & 11.82 \\
\hline 29 & 44 & 43.80 & 12 & 12.69 \\
\hline 30 & 44 & 44.51 & 11 & 10.47 \\
\hline 31 & 44 & 43.58 & 11 & 11.84 \\
\hline 32 & 43 & 42.51 & 11 & 11.49 \\
\hline 35 & 43 & 43.16 & 10 & 10.33 \\
\hline 36 & 43 & 42.56 & 10 & 10.33 \\
\hline 39 & 43 & 42.56 & 9 & 9.47 \\
\hline 40 & 43 & 43.22 & 9 & 9.49 \\
\hline 42 & 43 & 42.62 & 9 & 9.33 \\
\hline
\end{tabular}




\begin{tabular}{|c|c|c|c|c|}
\hline $\begin{array}{c}\text { Serial number of } \\
\text { the RV matrix }\end{array}$ & $\begin{array}{c}\text { Matrix row with } \\
\text { the highest value }\end{array}$ & $\begin{array}{c}\text { Estimated } \\
\text { Doppler } \\
\text { coordinate }\end{array}$ & $\begin{array}{c}\text { Matrix column } \\
\text { with the highest } \\
\text { value }\end{array}$ & $\begin{array}{c}\text { Estimated } \\
\text { distance } \\
\text { coordinate }\end{array}$ \\
\hline 46 & 43 & 42.49 & 8 & 8.13 \\
\hline 47 & 43 & 43.60 & 8 & 7.98 \\
\hline 49 & 43 & 43.27 & 7 & 7.84 \\
\hline 53 & 43 & 42.49 & 6 & 6.49 \\
\hline 54 & 43 & 42.51 & 6 & 6.13 \\
\hline 55 & 43 & 42.51 & 6 & 6.49 \\
\hline 56 & 43 & 43.33 & 6 & 6.18 \\
\hline 59 & 43 & 43.33 & 5 & 4.82 \\
\hline 60 & 43 & 43.82 & 5 & 5.18 \\
\hline 62 & 43 & 42.80 & 4 & 3.62 \\
\hline 63 & 43 & 42.67 & 4 & 3.62 \\
\hline 64 & 43 & 42.18 & 4 & 3.78 \\
\hline 65 & 43 & 43.60 & 3 & 2.82 \\
\hline 66 & 43 & 43.31 & 3 & 3.49 \\
\hline 67 & 43 & 43.47 & 3 & 3.18 \\
\hline
\end{tabular}

Note: False alarms were cleared from the table.

Source: The raw data the correction was made on was recorded in early 2020 at Budapest Ferenc Liszt International Airport.

\section{Tracker}

The tracker uses a UFIR-like algorithm to track the targets. The tracking is done in the bistatic plane. When a hit ingresses the tracker, it starts to track it using its bistatic velocity (calculated from the Doppler shift). When another hit comes close enough to the tracked one, it registers the second hit and changes the track state to established. From this moment on, the used tracker outputs a plot at every moment as a track.

\section{Theory of operation}

False alarm avoidance

Despite using every check and threshold selection described in the previous sections, false alarms can appear as inputs of the tracker. As every hit either initialises a new track or appears as a continuation of a current track, this could be dangerous; however, false alarms are quite random, hence plotting tracks having more than one hit appeared to be enough for this test. 


\section{Theory of tracking}

Tracking could be done in a Descartes plane in general. Doing so here is not a good choice, since the measuring is done in the bistatic polar plane, and the conversion from it to a Descartes one would have nonlinear distortion and nonlinear error distribution on it. This error would also appear in the conversion of the bistatic speed, which is a directly measured state variable of the system. The transformation of the speed has another difficulty as not every component can be directly calculated from the bistatic speed.

A solution is to do the tracking in the bistatic plane, then convert the track to a Descartes plane. The track should interpolate the hits; therefore, output points appear more frequently, so the information of the directly measured bistatic speed will be contained by the interpolated points on the classic plane.

Plots only mean samples from the plane's trajectory with some margin of error. Measuring its distance multiple times at the very same point cannot be accomplished in this test setup. One possible solution is using an unbiased FIR-like filter. In this setup, the sampling time is not consistent, because full RV matrices are dropped sometimes. Knowing that the bistatic acceleration is not constant in a real-world situation, this effect causes an accumulation of error in the UFIR. This could be decreased by a proper weight function.

Another mandatory information for the location is the angle of the arriving signal. This is measured with all the hits, but its first derivative is not given, as it was in the case of distance. The time function of the angle should also be estimated for the tracking. In these measures, the basic angular velocity formula was used and treated as a constant between hits.

\section{Tracking of the bistatic distance}

In the test, a three long FIFO was used to store the previously measured bistatic points with timestamps, and a separate point was used to track the head of the trajectory. Whenever there was a plot (a new output was generated), the oldest element of the FIFO was dropped, the new was introduced, and also the bistatic velocity of the newest point updated the state variable of all the points in the FIFO.

Every plot event updates every entry in the FIFO from their bistatic velocity (updated on every income) and the elapsed time from the last plot. The update is simply done by multiplying the velocity by the elapsed time, and then add the value to the original bistatic distance.

In the test environment, every element of the buffer was averaged with equal weight regardless of the difference of their income time, no time-adaptive weight function was used to produce a single distance coordinate for the output of the tracker. 


\section{Tracking of the angle}

Calculating the angle is different from the case of the bistatic distance since the derivative is not given. The method used to step this variable during plotting is to determine the angular velocity from the last two hits using the difference of the rotation and the time.

Although this could be a good solution, with an imaginary target moving on a constant bistatic curve with constant angular velocity, in real life, this scenario is not realistic. A target with a linear trajectory on a Descartes plane would fail the tracking - the tracker is unstable. Simulations prove this fact.

To stabilise the method mentioned above, a correction factor was used. At the point of the correction, the new plot's bistatic distance is calculated, but the angle is left in the previous state. Descartes coordinates of the previous and the current state are calculated. From these data, the static error of the tracking could be estimated analogue to the arcus tangent demodulation method in FM software radio systems:

$$
\operatorname{Corr}=\frac{\left(y \cdot x_{\text {before }}\right)-\left(x \cdot y_{\text {before }}\right)}{r_{\text {receiver }}^{2}}
$$

where $x$ and $y$ are the Descartes coordinates of the plot and the previous plot, and $r_{\text {receiver }}$ is the distance from the receiver for the currently calculated plot. The result is in radians. The correction (as the static error was integrated for the time between the before and the now state) should be subtracted from the new plot's angle, and after this, the previously calculated speed term should also be accumulated.

The correction method could be analogous to the FM demodulation technic because the set of the used points can be interpreted as a constellation diagram.

\section{Known limitations}

The bistatic speed is sampled with every hit, but since the trajectory of the target is mostly linear, the bistatic distance is not a linear function, hence the bistatic speed function as the first derivative of the distance is not sufficient for the tracking, the distance is either over or underestimated because of the zero order estimation on the sampled speed in the algorithm above. On average, this generates a rotated trajectory. In the future, a lookup method should be used, which could determine the speed from interpolated hits, then correct the coordinates from this information.

In some trajectories, crossing the perpendicular axis from the bistatic axis causes the loss of the tracking since the correction used in the angle calculation is failing, and cannot be used. 
Most of the computer systems used nowadays have parallel computing capability. Changing the used shortest path algorithm to another one utilising these resources may increase the speed of processing. ${ }^{7}$

\section{Testing}

To test the work of the tracker, a simulator was used. The blue points are the actual trajectory of the simulated target. The green points are the output of the tracker. The parameters used in the sample generator are shown in Figures 5, 6 and 7.

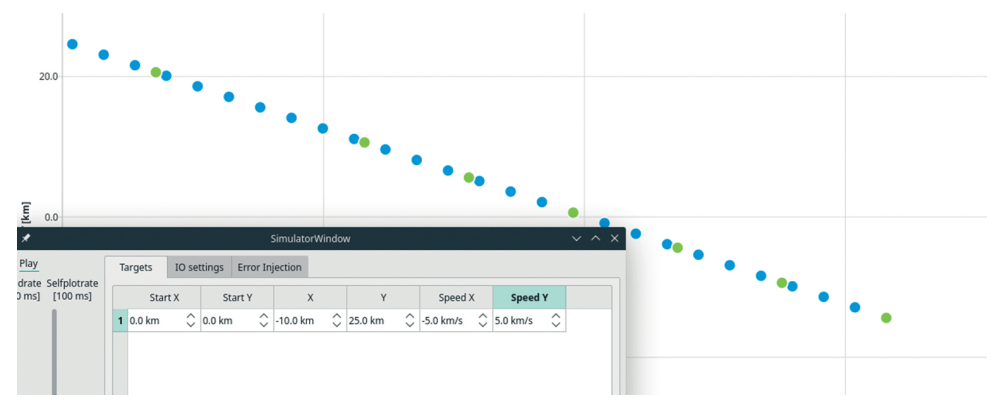

Figure 5: Output of the tracer on a simulation with linear, but not vertical trajectory

Source: The trajectories were generated using a simulator written for this test. Figure 5 simulates a coherent speed target with the shown parameters. Sent and received hits

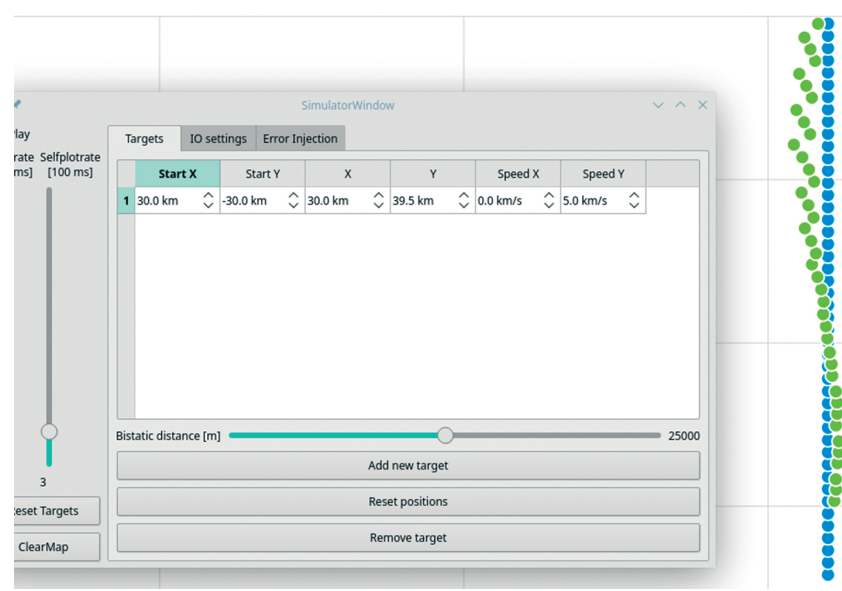

Figure 6: Output of the tracer on a simulation with linear and vertical trajectory

Source: The trajectories were generated using a simulator written for this test. Figure 6 simulates a coherent speed target with the shown parameters.

David R Alves, Madan S Krishnakumar and Vijay K Garg, 'Efficient Parallel Shortest Path Algorithms', 19th International Symposium on Parallel and Distributed Computing (ISPDC), Warsaw, 2020, 188-195. 


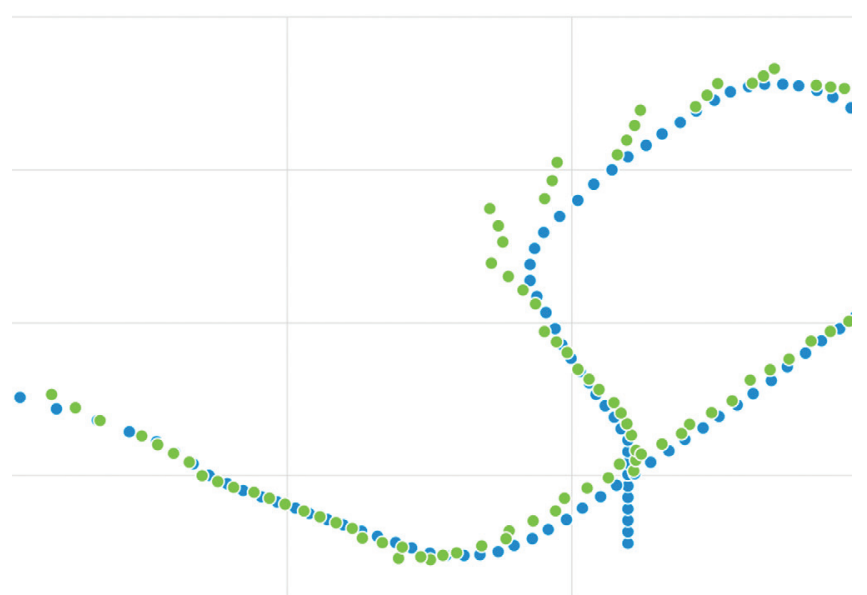

Figure 7: Output of the tracker on a simulation with nonlinear trajectory and additional Gaussian noise

Source: The trajectories were generated using a simulator written for this test. Figure 7 shows a dynamicly changing trajectory.

\section{Conclusion}

The proposed extractor tracker could be effectively used in FM-based passive radar systems for general ATC purposes. Targets having micro Doppler effect, such as helicopters, could appear on the RV matrices as more than one peak; therefore, they should be preprocessed to one point before using this tracking technique. The accuracy of the plots does not count in near distances, because in a system where multiple passive radars are used, more accurate measurements could be done with a directed signal processing on a wider band DVB-T or DVB-T2 channel. ${ }^{8}$ DVB passive radars consume more power than an FM one since due to its wider spectrum occupation, more processing cycles are required to produce a result. By only processing a smaller part of space and a smaller range of Doppler shifts, the terrestrial DVB passive radar uses much less computation power. Having more FM channels as reference sources, compressed sensing technology could be used to produce a higher quality of RV matrices. In a more complex tracking, for example having inputs from more illuminators or targets, better state estimation technics could be done, such as a particle filter.

8 Rudolf Seller, Tamás Petô, Levente Dudás and Levente Kovács, 'Passzív radar II. rész', Haditechnika 54, no 1 (2020), 43-47. 


\section{References}

Alves, David R, Madan S Krishnakumar and Vijay K Garg, 'Efficient Parallel Shortest Path Algorithms'. 19th International Symposium on Parallel and Distributed Computing (ISPDC), Warsaw, 2020, 188-195. Online: https://doi.org/10.1109/ ISPDC51135.2020.00034

Bo, Li and Sun Chao, 'A new method for array extension based on minimum redundancy linear array’. IEEE 10th International Conference on Signal Processing Proceedings, Beijing, 2010, 332-335. Online: https://doi.org/10.1109/ICOSP.2010.5654981

Meller, Michal and Kamil Stawiarski, 'Capon-like DoA estimator for rotating arrays’. 2019 International Radar Conference (RADAR), Toulon, 2019, 1-6. Online: https://doi. org/10.1109/RADAR41533.2019.171281

Peng, Chen, Xiaoqing Lu, Jiyang Dai and Linfei Yin, 'Research of path planning method based on the improved Voronoi diagram'. 25th Chinese Control and Decision Conference (CCDC), Guiyang, 2013, 2940-2944. Online: https://doi.org/10.1109/ CCDC.2013.6561448

Seller, Rudolf, Tamás Pető, Levente Dudás and Levente Kovács, 'Passzív radar I. rész'. Haditechnika 53, no 6 (2019), 51-55. Online: https://doi.org/10.23713/HT.53.6.10

Seller, Rudolf, Tamás Pető, Levente Dudás and Levente Kovács, 'Passzív radar II. rész’. Haditechnika 54, no 1 (2020), 43-47. Online: https://doi.org/10.23713/HT.54.1.09 